\title{
Management and Reconfiguration of a Radiology Department under the Threat of Coronavirus Disease 2019: Experience from Wuhan
}

\author{
Meng TIAN ${ }^{1 \dagger}$, Cong-cong LIU ${ }^{1 \dagger}$, Lu LONG ${ }^{2 \#}$, Qiu-xia WANG ${ }^{1 \#}$ \\ ${ }^{1}$ Department of Radiology, Tongji Hospital, Tongji Medical College, Huazhong University of Science and Technology, Wuhan \\ 430000, China \\ ${ }^{2}$ Department of Epidemiology and Biostatistics, West China School of Public Health, Sichuan University, Chengdu 610000, \\ China
}

(C) Huazhong University of Science and Technology 2020

\begin{abstract}
Summary: The corona virus disease 2019 (COVID-19) is an emerging respiratory infectious disease caused by SARS-CoV-2, which first occurred in December 2019 in Wuhan, China. These days, in China, chest CT is used for diagnosis of COVID-19, as an important complement to the reverse-transcription polymerase chain reaction (RT-PCR) test. Because of contacting with a large number of suspected or probable cases closely during chest CT examination, radiographers are easily infected with COVID-19. This article included the rearrangement of CT examination room in fever clinic, the rearrangement of human resources in radiology department, and the drafting of new operating procedures for radiologists who carry out CT examination on COVID-19 patients. This article also introduced the emergency management procedures of the department of radiology during the outbreak, and the experience of infection prevention for the staff of the department of radiology.
\end{abstract}

Key words: COVID-19; management; reconfiguration; radiology department

In December 2019, a new viral pneumonia case occurred in Wuhan, Hubei Province. Because it is a respiratory disease that has never been experienced before and has a wide and rapid infection ability, it has attracted the attention of all countries in the world ${ }^{[1,2]}$. High-throughput sequencing has revealed a novel beta coronavirus, which is similar to severe acute respiratory syndrome coronavirus (SARS-CoV) ${ }^{[3]}$. As of March 2020, 193475 confirmed human cases of infection with corona virus disease 2019 (COVID-19) including 7864 deaths had been reported from 164 countries $^{[4]}$.

Patients with uncomplicated upper respiratory tract viral infection, may have non-specific symptoms such as fever, fatigue and dry cough. The diagnosis of COVID-19 is based on epidemiological history, clinical manifestation and laboratory examination results. Routine confirmation of cases of COVID-19 is based on detection of unique sequences of virus RNA by nucleic acid amplification tests (NAAT) such as real-time reverse transcription polymerase chain reaction (rRT-PCR) with confirmation by nucleic acid

Meng TIAN, E-mail: tianmengfsk@163.com; Cong-cong LIU, E-mail: liucongcongfsk@163.com

${ }^{\dagger}$ The authors contributed equally to this work.

\#Corresponding authors, Lu LONG, E-mail: longlu201609@, 163.com; Qiu-xia WANG, E-mail: wangqiuxia@hust.edu.cn sequencing when necessary ${ }^{[5]}$.

However, with the limitations of sample collection, transportation, and kit performance, the total positive rate of RT-PCR for throat swab samples was reported to be approximately $30 \%$ to $60 \%$ at the initial presentation. Meanwhile radiographic studies showed that the majority of viral pneumonias had similar features on CT images, like ground glass opacity (GGO) or mixed GGO and consolidation. COVID-19 is likely to have a peripheral distribution with bilateral, multifocal lower lung involvement. In this emergency situation, the "Guideline of diagnosis and treatment of pneumonitis caused by COVID-19 (trial fifth version)" was released by the Chinese National Health Commission ${ }^{[6]}$, adding a clinical diagnosis classification in Hubei Province and taking chest imaging features as one of the clinical diagnostic criteria. With the spread of the pandemic, the radiology department is facing increasingly heavy inspection tasks and high standards of protection requirements. The standardized scanning process and rigorous protective measures allow the majority of technicians to complete patient examinations while reducing the risk of occupational exposure during the pandemic.

Tongji Hospital is one of the hospitals with the largest number of COVID-19 patients in Wuhan. More than 1500 inpatients were diagnosed here. This 
article summarized the effective measures taken by the Radiology Department of Tongji Hospital to address the epidemic.

\section{HOSPITAL COVID-19 PROTECTION CONTROL PLAN}

In the early stage of the outbreak, we took positive measures to stop general outpatient services and set aside a special area of the outpatient service as the fever outpatient service, establishing a partitioned, isolated area within the general outpatient service and emergency department. The fever ward was equipped with an independent nurse station, observation area, isolation rooms, designated elevator, CT examination room, etc. The observation area was for receiving suspected, probable patients, while negative pressure isolation rooms were designated for confirmed patients, and each patient was isolated within a single room. The department, which is responsible for patient transport, designated a specific elevator for COVID-19 patients to reduce the risk of cross infection. To prevent hospital staff and nonCOVID-19 patients from being infected, the hospital strengthened the pre-examination and triage of the patients and implemented the three-level triage. The fever-screening procedure for suspected patients is indicated in fig. 1.

\section{EMERGENCY MANAGEMENT AND INFEC- TION CONTROL IN THE RADIOLOGY DEPAR- TMENT}

Due to the fast speed, high sensitivity and strong specificity of CT examinations, almost every suspicious patient should receive a CT examination. Because technicians have direct communication and contact with patients in the examination process, the risk of cross infection is greatly increased. Therefore, on the basis of the hospital's instructions, the radiology department took strict control measures, strengthened prevention accordingly, and made systematic personnel arrangements and adjustments to minimize the possibilities of medical staff infection and other cross infections. This article elaborates on a series of prevention and control measures and principles adopted by the Radiology Department of Tongji Hospital during the outbreak stage.

2.1 Establishment of Infection Control Team in Radiology Department and Staff Risk Level Grading

In the early stage of the outbreak, the Radiology Department quickly established an infection control team and graded the risk level (table 1) according to the

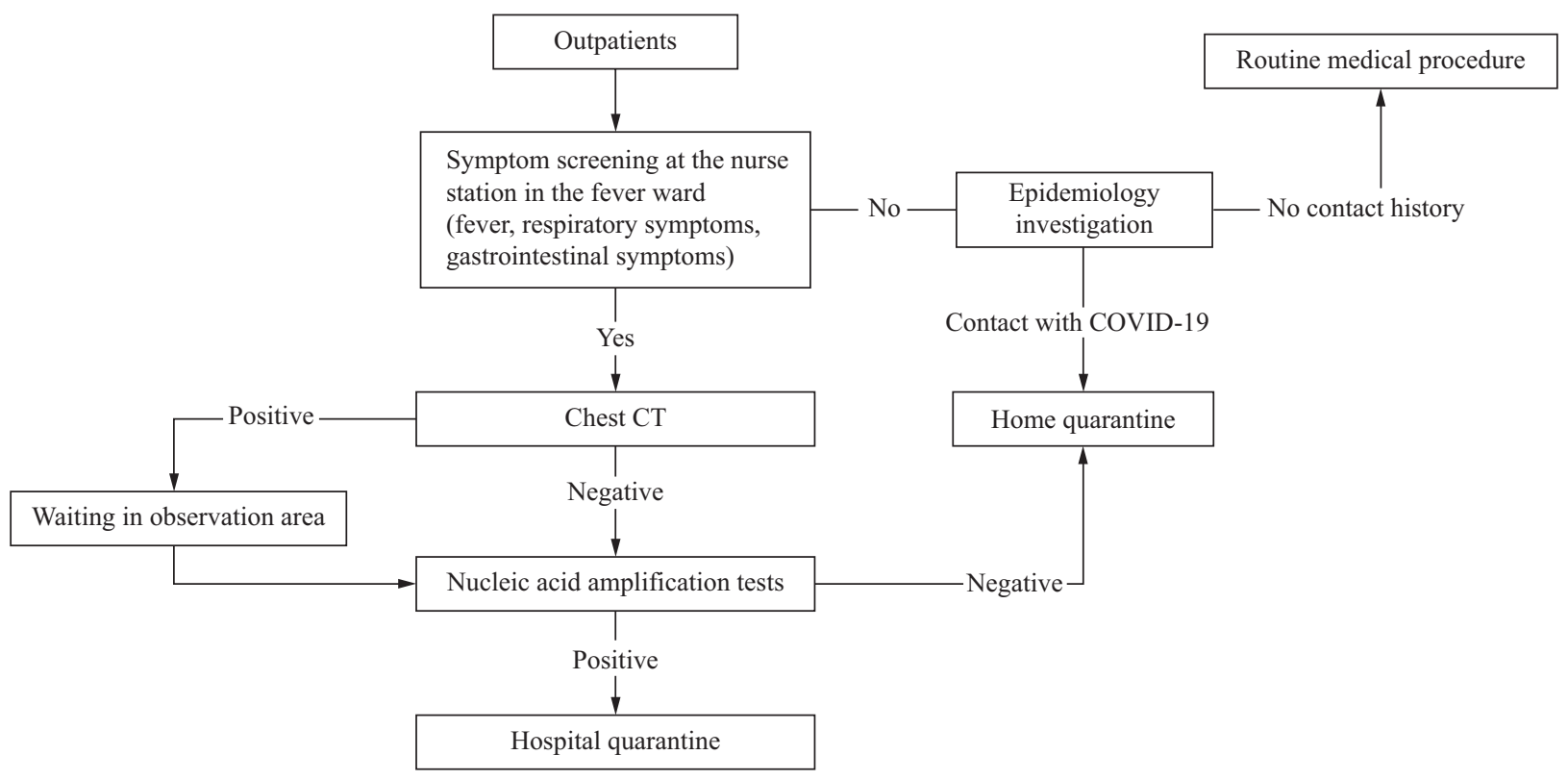

Fig. 1 Screening procedures of suspected cases at Tongji Hospital

Table 1 Risk of the team members

\begin{tabular}{ll}
\hline Risk classification & Team members involved \\
\hline Ultra-high risk & $\begin{array}{l}\text { People who have direct contact with suspected or confirmed patients infected with COVID-19, such as CT } \\
\text { technicians, bedside technicians in fever ward, etc. } \\
\text { High risk }\end{array}$ \\
$\begin{array}{l}\text { People having contact with the suspected or confirmed patient's environment directly, such as technicians } \\
\text { performing routine CT and cleaners in CT room }\end{array}$ \\
$\begin{array}{l}\text { People who are in contact with the screened infected person, such as registrars, MRI and Dr examinations, nurses. } \\
\text { Low risk }\end{array}$ & People who are not in contact with patients or contaminated areas, such as radiologists, managers, etc. \\
\hline
\end{tabular}


nature of the team members' work and the workplace to ensure that the risk of cross-infection was minimized while the team members were completing their work. The infection control team consists of administrators, radiologists, technicians, nurses, registrars and others. The main responsibilities of the infection control team include adjusting the setting of the special CT room for patients with fever, coordinating the staffing of the Radiology Department, reasonably planning the examination process for patients with fever, coordinating the configuration of protective equipment, and sterilizing the room and other areas.

\subsection{Layout of Radiology Department}

COVID-19 is infectious and easily causes crossinfection. The Radiology Department thus opened a special independent CT room in the fever outpatient service area to separate patients with fever from other patients. As it is not connected to other CT rooms, the independent CT room is equipped with a remote sensing protective lead door and CT machine tool lift that can be remotely controlled. To reduce the possibility of cross-infection, attention must be paid to the use of central air conditioning. At the same time, the following areas must be set up: a contaminated area, semicontaminated area, buffer area and clean area. The contaminated area mainly includes the CT examination room, waiting area and passageway for patients with fever. The semicontaminated area mainly includes the CT operating room. The buffer area mainly includes the passageway between the semicontaminated area and the dressing room, and the clean area mainly includes the dressing room, office, rest room, diagnosis reading room and warehouse. In addition, a location was designated for printing the results of fever patients (fig. 2).

2.3 Reorganization of CT Technician Staffing

To minimize the possibility of cross-infection, CT

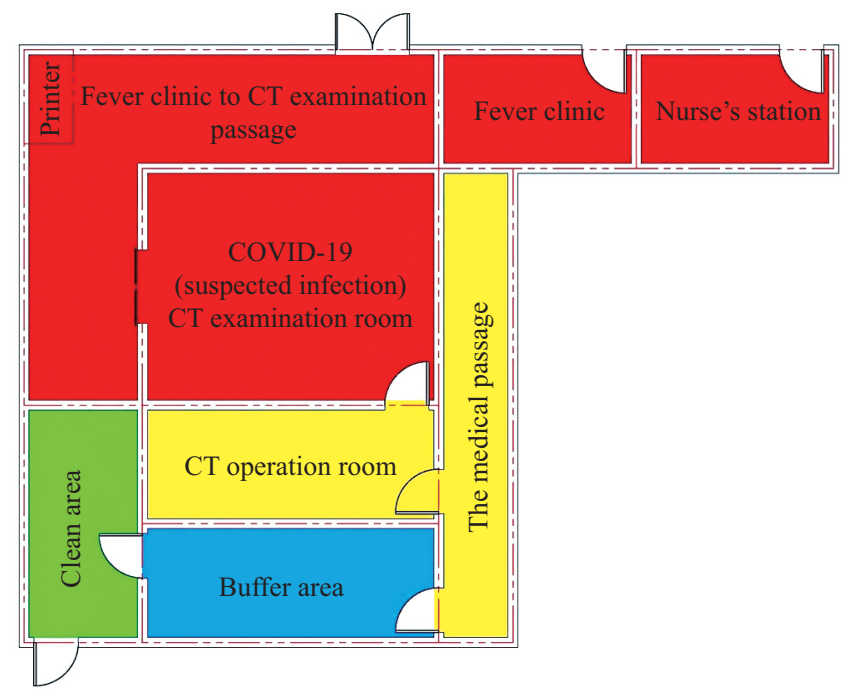

technicians in the fever clinic should be reconfigured to ensure the normal operation of other departments, and the technicians involved in CT work concerning patients with fever should be relatively fixed. Priority should be given to technicians with a strong physical condition, no underlying diseases, and experience in dealing with similar emergent infectious diseases. Before entering the fever clinic, pre-job protection training for all staff should be provided. One CT device is best equipped with two staff members who are responsible for the operation and positioning, and the contact between the operator and positioning personnel should be minimized to ensure the least possible contamination in the operating room. In addition, family members, caregivers and clinicians of patients with fever are prohibited from entering the operating room. For the technicians involved in the fever clinic, a "short time on duty, long time quarantine" policy is applied. In addition, psychological counselors or special channels for psychological counseling are arranged to conduct mental health counseling, evaluations and education for staff participating in CT examinations in fever clinics.

\subsection{CT Examination Procedure and Operation Specification}

CT examinations of patients with fever should follow the principle of "come and go" to shorten the time that patients are in the Radiology Department. CT examinations of patients in the fever ward are led and managed by a specific person and completed intensively at an appropriate time (fig. 3).

2.4.1 Pre-inspection Preparation The technicians perform personal protection as required, preheat the CT device and complete the calibration to ensure that the machine and the network are functioning well. The technicians should lower the examination bed to the appropriate height in advance and place a separate

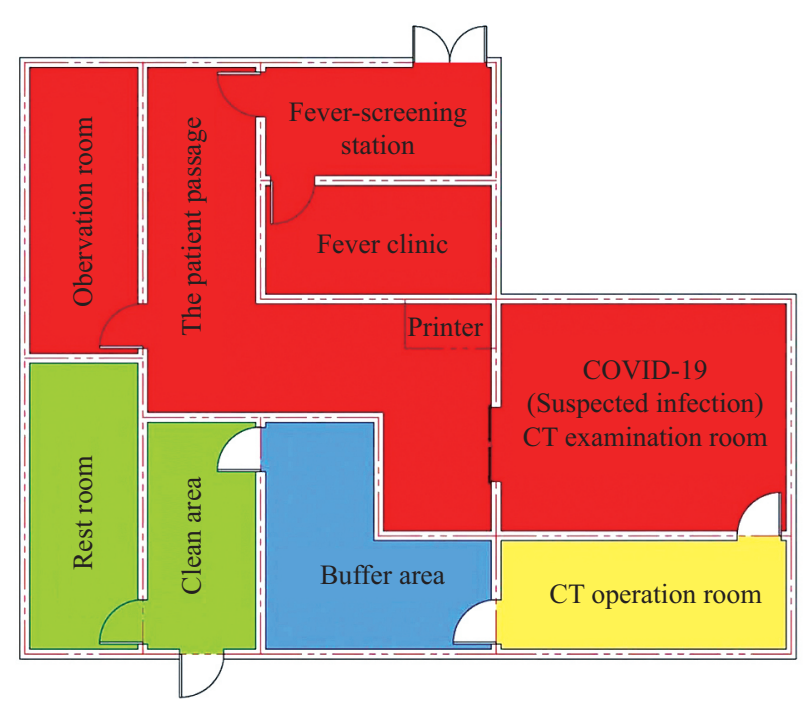

Fig. 2 Diagram of the layout of our radiology department of fever clinic (not to scale) showing barriers that limit the movement of patients to the imaging rooms 


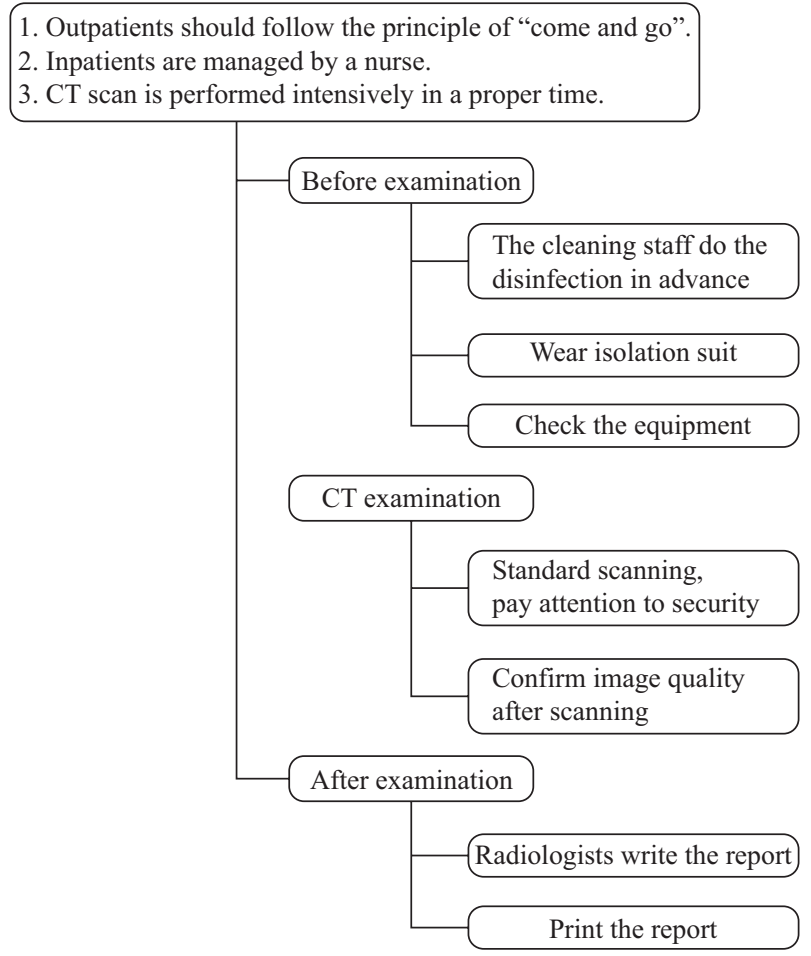

Fig. 3 Flow chart of CT examination of patients with COVID-19

sheet (the sheets are changed for each patient) on the examination bed to reduce equipment contamination. The technicians should also call the patient's orderly through the station-to-station system to prepare them in advance and inform the patients and their families to wear masks at all times.

2.4.2 Operation Specifications After the patient enters the CT examination room, the technician must check the patient's information remotely, confirm the examination items and special requirements, and tell the patient to remove metal ornaments from his/her neck and chest. On the premise of ensuring safety, patients should be told to lie down on the examination bed and raise their hands; the patients should also be trained to hold their breath at the end of inhalation in advance. Those who find it difficult to perform the breath training should be told to only hold their breath. For patients with a disturbance of consciousness or who are unable to take care of themselves, they may ask their accompanying family members or technicians responsible for positioning to help them achieve the required position. After reaching the required position, the technician should perform additional positioning. The normal positioning button should be covered with a transparent film to ensure that the technician does not have direct contact with the machine. Considering that the patient may undergo multiple $\mathrm{CT}$ examinations in the later stages, the appropriate scanning protocol should be selected to avoid harmful biological effects on the human body caused by multiple examinations in the short term.
2.4.3 Scanning Range and Parameters The spiral scanning method is used to turn on the automatic tube voltage or choose a tube voltage of $100-120 \mathrm{kV}$ and intelligent milliampere-second of 50-350 mAs. The width of the collimator is $0.5-1.5 \mathrm{~mm}$, of which the layer thickness and the layer spacing are $1-5 \mathrm{~mm}$. For patients with severe or critical symptoms, a larger pitch $(1.0-1.5$ pitch $)$ is used to reduce the scanning time and reduce the patient's respiratory movement artifact. The scanning direction and range are from the tip of the lung to the costophrenic angle, and the scanning direction from the costophrenic angle to the tip of the lung can be adopted to reduce the artifact of respiratory movement for patients with dyspnea.

2.4.4 Post-inspection Specifications After the patient has completed the scan, the technician ensures that the image quality meets the diagnostic criteria before the end of the examination. Then, the technician lowers the examination bed to the appropriate height and tells the patient to leave. After the technician confirms that the image has been uploaded automatically, he or she calls the radiologist to write the report and prints the film in a timely manner.

It is worth noting that screening patients with no typical symptoms and with a clear epidemiological history (these patients are generally examined in the general CT examination room) is an important means of avoiding cross-infection during a new coronavirus outbreak. Patients with no typical symptoms but highly suspicious chest $\mathrm{CT}$ images should be immediately sent to the fever clinic for further screening, and the air, objects and floor of the operating room and machine room should be disinfected after the examination of such patients.

\subsection{Bedside Examination of Critically III Patients}

For some COVID-19 patients who cannot leave the equipment of the ward for a CT examination, the clinician will perform a bedside DR examination. The mobile bedside DR for critically ill patients should be equipped with a special device in each ward to prevent the machine from being pushed back and forth and contaminating the hospital area, and the time of bedside examination should be concentrated as much as possible to avoid frequent contact of technicians with patients (except during an emergency). After receiving notice, the technicians should take personal protection precautions before entering the isolation ward, start the equipment, connect to the network, and collect and check the patient information. Before photographing, the detector should be protected by a special protective cover with 1-2 layers. Detector placement should be assisted by others for critical patients who cannot turn over. The photographing distance should be adjusted, the $\mathrm{kVs}$ and $\mathrm{mAs}$ should be increased, and delayed exposure should be opened. After photographing, the image quality should be confirmed, the image should 
be transmitted to PACS, and the protective cover of the detector should be removed to ensure that the contaminated surface does not touch other objects. After finishing the operation, technicians must disinfect their hands, push the equipment back to the specified area and call the cleaning staff to disinfect the machine and its activity area (fig. 4).

2.6 Radiology Staff Protection Standards (Table 2) and Equipment Disinfection Requirements (Table 3)

Personal protection is performed by technicians in accordance with the second level of protection ${ }^{[7-9]}$. The use of a medical disposable cap, goggles, face guard (anti-fog type), mask, disposable gown, protective coverall, gloves, and shoe covers as well as proper hand hygiene are strictly enforced. A quick hand cleansing should be carried out before and after inspection. Nurses should avoid contact with the patient's blood or bodily fluids as much as possible and conduct rapid hand cleansing before and after contact. Radiologists should perform personal protection according to the first level of protection and communicate with technicians and patients via communication equipment as much as possible. Radiologists should not enter contaminated areas or semicontaminated areas and shall not have direct contact with patients. When emergency treatment is required in a medical

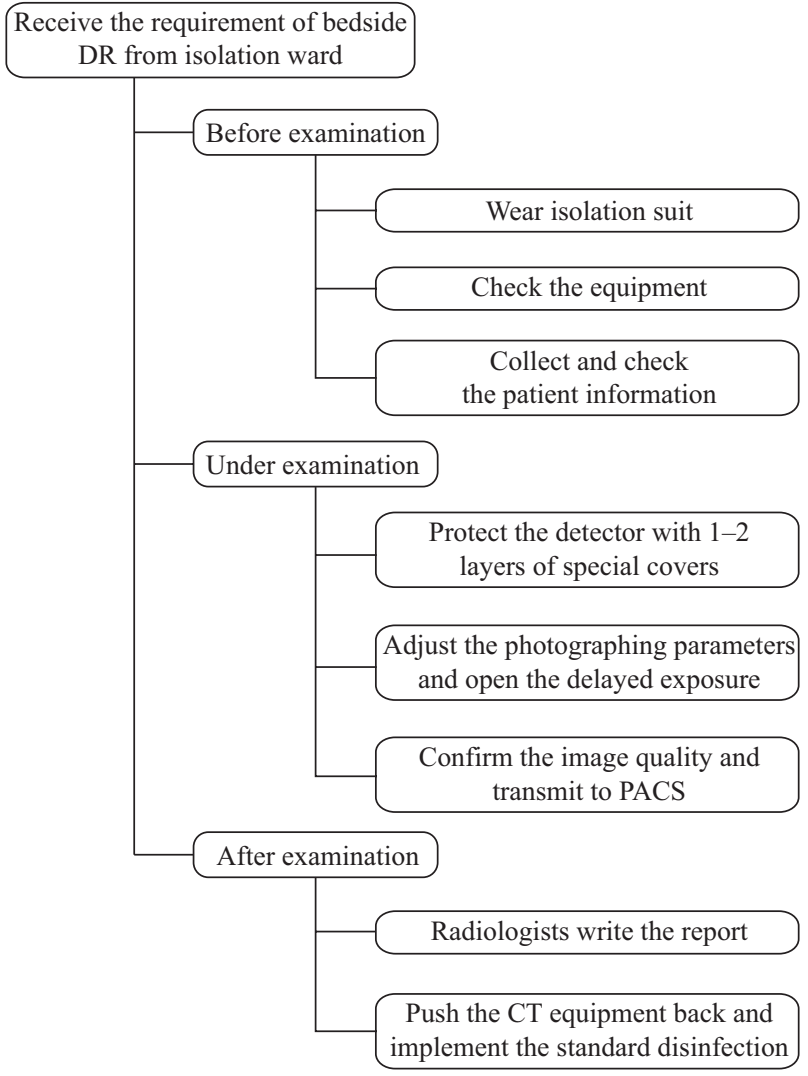

Fig. 4 Flow chart of bedside examination of critically ill patients with COVID-19

Table 2 Safety equipment requirements for radiology staff according to different risk classification specifications

\begin{tabular}{lcccc}
\hline Items & Ultra-high risk & High risk & Medium risk & Low risk \\
\hline Mask & N95+surgical & N95 & N95 & Surgical \\
Gloves & 2 sets & $\sqrt{ }$ & $\sqrt{ }$ & V \\
Medical disposable hat & 2 sets & $\sqrt{ }$ & $\sqrt{ }$ & $\bullet$ \\
Goggles & $\sqrt{ }$ & $\sqrt{ }$ & $\bullet$ & $\bullet$ \\
Face guard & $\sqrt{ }$ & $\sqrt{ }$ & $\sqrt{ }$ \\
Disposable gown & $\sqrt{ }$ & $\bullet$ & $\bullet$ \\
Disposable protective coverall & 2 sets & $\sqrt{ }$ & \\
Shoe covers & l & & $\sqrt{ }$ & \\
\hline
\end{tabular}

$\sqrt{ }$ necessary item; $\bullet$ worn according to condition

Table 3 Disinfection of common contaminated object

\begin{tabular}{|c|c|}
\hline Contaminated object & Method of disinfection \\
\hline Air & $\begin{array}{l}\text { CT examination room: sterilize with UV irradiation for } 60 \mathrm{~min}, 4 \text { times a day, without people around; } \\
\text { Other areas: spray } 1000 \mathrm{mg} / \mathrm{L} \text { chlorine-containing disinfectant }\end{array}$ \\
\hline Surface & $\begin{array}{l}\text { Normal: clean with } 1000 \mathrm{mg} / \mathrm{L} \text { chlorine-containing disinfectant; } \\
\text { Non-corrosion-resistant surfaces: wipe with } 75 \% \text { ethanol twice; } \\
\text { Visible contamination: remove the pollutants completely with disposable absorbent material, and then cover } \\
\text { with } 2000 \mathrm{mg} / \mathrm{L} \text { chlorine-containing disinfectant for } 30 \text { min before being used for wiping and disinfection }\end{array}$ \\
\hline Equipment & $\begin{array}{l}\text { Equipment in the contaminated area: clean with } 2000 \mathrm{mg} / \mathrm{L} \text { chlorine-containing disinfectant (the DR or CT rack } \\
\text { should be cleaned with } 75 \% \text { ethanol) twice a day; } \\
\text { Equipment in a common machine room: clean with } 500-1000 \mathrm{mg} / \mathrm{L} \text { chlorine-containing disinfectant or with } \\
\text { disposable disinfection wipes containing alcohol twice a day; } \\
\text { In cases of contamination, disinfecting procedures should be performed at any time. }\end{array}$ \\
\hline Floor & $\begin{array}{l}\text { Wipe and disinfect the floor with } 1000 \mathrm{mg} / \mathrm{L} \text { chlorine-containing disinfectant once every } 4 \mathrm{~h} \text {; } \\
\text { In cases of contamination, disinfecting procedures should be performed at any time. }\end{array}$ \\
\hline Hand hygiene & $\begin{array}{l}\text { Perform hand hygiene procedures in strict accordance with the "two before and three after" principle (before: } \\
\text { before contact with patients, before aseptic operation; after: after bodily fluid exposure, after contact with the } \\
\text { patient, after contact with the patient's surrounding environment), and both before and after wearing isolation } \\
\text { clothing, protective clothing and gloves }\end{array}$ \\
\hline
\end{tabular}


accident, radiologists should upgrade to the second level of protection. CT equipment and the inspection environment should be checked strictly in accordance with the "Technical Specifications for Disinfection of Medical Institutions", "Management Specifications for Cleaning and Disinfection of Environmental Surfaces of Medical Institutions" and "Standards for Disinfection and Hygiene of Hospitals" to ensure the complete disinfection of medical instruments, contaminated articles, floors, air, etc.

\section{CONCLUSION}

COVID-19 is highly pathogenic and infectious. Early diagnosis of COVID-19 patients is of great significance to avoid cross infection. CT examinations play an important role in the confirmation of COVID-19. Therefore, in the crucial stage of the outbreak, the radiology department as an auxiliary diagnosis unit must quickly make targeted measures to ensure that the work is completed while the risk of infection is reduced.

First, immediately setting up a special prevention and control panel is necessary, which helps classify staff risks and helps them work more efficiently and with a clear protective principle. Reorganizing the layout of the CT devices in the fever outpatient clinic is an important measure to eliminate the routes of transmission. Strict implementation of the layout principle of "three areas, two passages" is the key to reduce the cross infection.

Second, a specific schedule and isolation measures not only protect technicians' physical health but also reduce the mental pressure on technicians. For technicians who carry out examinations for clinically confirmed patients, it is particularly important to strengthen the awareness of protection and pay attention to the protective measures of inspection equipment to protect themselves and to avoid cross-infection among patients. In brief, the standardized inspection process guarantees low-risk, efficiency and accuracy.

Third, technicians should master protection and disinfection procedures to reduce the risk of nosocomial infection. Each individual involved in a radiation inspection must be clearly informed of clean areas, contaminated areas and semicontaminated areas, preventing clean areas from being infected. Technicians must get changed and complete the handover in the specified location, strictly implement hand hygiene before and after the inspection, disinfect each area timely according to the national standards.

The COVID-19 outbreak is a challenge for both the hospital and the radiology department. It is the core task of the radiology department to quickly implement reasonable prevention and control measures and work arrangements. The measures introduced in this article provide previous experience and methods for future use when encountering similar sudden outbreaks.

\section{Acknowledgments}

We acknowledge all the staff who are at the forefront of the fight against the COVID-19 in the radiology department; We also thank all health-care workers involved in the diagnosis and treatment of patients in our hospital.

\section{Conflict of Interest Statement}

We declare that we have no conflicts of interest.

\section{REFERENCES}

1 WHO. Clinical management of severe acute respiratory infection (SARI) when COVID-19 disease is suspected, 2020.

2 Novel Coronavirus Pneumonia Emergency Response Epidemiology T. The epidemiological characteristics of an outbreak of 2019 novel coronavirus diseases (COVID-19) in China. Zhonghua Liu Xing Bing Xue Za Zhi (Chinese), 2020,41(2):145-151

3 Wrapp D, Wang N, Corbett KS, et al. Cryo-EM structure of the 2019-nCoV spike in the prefusion conformation. Science, 2020,367(6483):1260-1263

4 WHO. Novel Coronavirus (COVID-19) Situation. 2020, Available from: https://experience.arcgis.com/ex perience/685d0ace521648f8a5beeee $1 \mathrm{~b} 9125 \mathrm{~cd}$.

5 WHO. Laboratory testing for coronavirus disease 2019 (COVID-19) in suspected human cases, 2020.

6 New coronavirus pneumonia prevention and control program (7th ed) (Chinese). 2020,33.

7 Technical guidelines for hospital infection control of influenza A (H1N1) (Chinese). 2009.

8 Technical guidelines for prevention and control of human infection with swine influenza (Chinese). 2009.

9 Prevention and control of nosocomial infection by air borne diseases (Chinese). 2016.

(Received Apr. 28, 2020; accepted June 24, 2020) 